\title{
Numerical simulations of enhanced ion current losses in the inner magnetically insulated transmission line of the $Z$ accelerator
}

\author{
D. V. Rose $\odot,{ }^{1,}{ }^{*}$ E. M. Waisman, ${ }^{2}$ M. P. Desjarlais, ${ }^{2}$ M. E. Cuneo, ${ }^{2}$ B. T. Hutsel, ${ }^{2}$ D. R. Welch, ${ }^{1}$ \\ N. Bennett, ${ }^{2}$ and G. R. Laity ${ }^{2}$ \\ ${ }^{1}$ Voss Scientific, LLC, Albuquerque, New Mexico 87108, USA \\ ${ }^{2}$ Sandia National Laboratories, Albuquerque, New Mexico 87185, USA
}

(Received 24 March 2020; accepted 31 July 2020; published 14 August 2020)

\begin{abstract}
Two-dimensional electromagnetic (EM) particle-in-cell (PIC) simulations of a radial magneticallyinsulated-transmission-line are presented and compared to the model of E. M. Waisman, M. P. Desjarlais, and M. E. Cuneo [Phys. Rev. Accel. Beams 22, 030402 (2019) in the "high-enhancement" (WDC-HE) limit. The simulations use quasi-equilibrium current and voltage values based on the Sandia National Laboratories $Z$ accelerator, with prescribed injection of an electron sheath that gives electron density profiles qualitatively similar to those used in the WDC-HE model. We find that the WDC-HE model accurately predicts the quasiequilibrium ion current losses in the EM PIC simulations for a wide range of current and voltage values. For the case of two ion species where one is magnetically insulated by the ambient magnetic field and the other is not, the charge of the lighter insulated species in the anode-cathode gap can modify the electric field profile, reducing the ion current density enhancement for the heavier ion species. On the other hand, for multiple ion species, when the lighter ions are not magnetically insulated and are a significant fraction of the anode plasma, they dominate the current loss, producing loss currents which are a significant fraction of the lighter ion WDC values. The observation of this effect in the present work is new to the field and may significantly impact the analysis of ion current losses in the $Z$ machine inner MITL and convolute.
\end{abstract}

DOI: 10.1103/PhysRevAccelBeams.23.080401

\section{INTRODUCTION}

Experimental measurements and numerical simulations suggest significant current losses in the inner magnetically insulated transmission line (MITL) of the $Z$ accelerator [1-4] at Sandia National Laboratories, although direct measurements of these losses has not been obtained to date. In a recently published paper [5], a model for enhanced ion current losses in the inner MITL of $Z$ [6-8] was presented. The model assumes that vacuum electron flow close to the anode enhances the electric field, thereby increasing the realizable ion current emitted from the anode which results in ion current loss enhancements well in excess of the nominal Child-Langmuir (CL) limit for ions. The sheath flow pattern in which electrons flow along the anode surface is a result of the current adding convolute coupled to the inner radial MITL on $Z$ (see, for example, Ref. [2] and references therein).

\section{*David.Rose@vosssci.com}

Published by the American Physical Society under the terms of the Creative Commons Attribution 4.0 International license. Further distribution of this work must maintain attribution to the author(s) and the published article's title, journal citation, and DOI.
As discussed in Ref. [5], the enhancement of ion current with respect to the $\mathrm{CL}$ ion-only law, due to the presence of an $E \times B$ electron flow charge in the anode-cathode (AK) gap, goes back to the work of Desjarlais [9] for applied magnetic field ion diodes. In that research the saturated flow case is defined, such that for the appropriate constant electron charge density filling the gap, the enhancement factor $\eta=j_{i} / j_{\mathrm{CL}}$, where $j_{i}$ is the ion current density and $j_{\mathrm{CL}}$ is the CL ion current density given in Eq. (1) below. Electron charge distributions which produce $\eta$ higher than this value are discussed, including the case resulting in $\eta \rightarrow \infty$. In the context of trying to explain ion current loss in the convolute and inner MITL of the $Z$ machine, this possibility was examined first by Hutsel et al. [10]. In [10] a detailed transmission line circuit code for $Z$ is introduced and employed as a tool to fit and predict $Z$ experiment results. The code computes the electron sheath current entering from the outer MITLs into the convolute, employing fitting parameters for the fractions of that current lost and accumulated in the convolute and inner MITL regions. Upon formation of anode plasma, the ion loss current of nonmagnetically insulated ions is calculated as a function of the time integral of the inner MITL surviving electron sheath current and local voltage and gap. In the Appendix D a comparison between that formulation and 
that of Ref. [5] is provided. We remark that neither Ref. [5] nor Ref. [10] address the interaction between magnetically and nonmagnetically insulated ion species, which is indeed one of the main subjects of this paper.

The simulations presented here do not include the development of electrode plasmas. Thus the simulations are based on the assumption that the development of electrode plasmas in the inner MITL only enters by permitting space-charge-limited (SCL) emission of charged particles and determining the effective AK gap. Our considered dynamics pertains to noncollisional plasma physics, both for electrons and ions. Since we only consider steady state (fixed AK gaps) our present work ignores the impact of electrode plasma expansion. Recent simulation studies [11,12] offer an additional current loss mechanism, partially based on identifying turbulently driven plasma transport in the inner MITL. As we discuss in Sec. VII, pulsed power experiments are needed to determine the main current loss mechanisms in the inner MITL. In Sec. VII we also discuss the implications of our present simulations, having shown the dependence of ion current loss with voltage, current, and AK gap.

A series of two-dimensional (2D), fully electromagnetic (EM) particle-in-cell simulations using CHICAGO [13] are compared directly with the model of Waisman, Desjarlais and Cuneo [5] in the "high-enhancement" (WDC-HE) limit. The simulations are operated in a quasi-equilibrium limit for easier comparison to the model of Ref. [5]. Furthermore, the simulations are executed in a 2D radial (parallel-plate) configuration for operating parameters that are similar to those found in the inner MITL of the $Z$ accelerator.

Overall we find that the simulations of a single nonmagnetically insulated (NMI) ion species support the WDC-HE model in the presence of a high-current (order $0.5 \mathrm{MA}$ ) electron sheath propagating close to the anode. Although the simulations exhibit large fluctuations due to the nonlinearly evolving electron sheath propagating through the inner MITL and ion emission region, temporally and spatially averaged ion current loss values from these simulations agree well with the WDC-HE model for a single ion species.

Simulations that include two ion species (here $\mathrm{H}^{+}$and $\mathrm{O}^{+}$) emitted from the anode can exhibit a shielding characteristic when the lighter species is magnetically insulated (MI) from striking the cathode, which in turn reduces or prevents the heavier NMI species from striking the cathode as well. Using a new, modified form of the WDC-HE model to partially account for the mix of ion species, approximate agreement with the multiple ion species emission simulations is found.

In Sec. II, the 2D EM simulation model used in this work is described. In Sec. III, the baseline simulation result is described in some detail and in Sec. IV, additional single ion species simulations are presented that include a number of parameter variations. In Sec. V, 1D electrostatic simulations of single and multiple SCL ion currents are described that verify the WDC-HE model in the case of a single ion species, and illustrate the validity of the model when applied to two ion species that are NMI or partially magnetically insulated (PMI). In Sec. VI, we describe 2D EM simulations where two ion species are used with either a dynamically injected electron sheath or a static electron density profile preloaded in the simulation. A summary and a discussion of the next steps for theoretical study and experimental measurements are given in Sec. VII. Finally, Appendixes describing the electron sheath properties used in the 2D simulations and the impact of spatial resolution and macroparticle counts on the results of the 2D EM simulations are given.

\section{NUMERICAL SIMULATION MODEL}

The baseline 2D simulation model assumes a radial vacuum transmission line extending from $r=11 \mathrm{~cm}$ to $r=3.5 \mathrm{~cm}$; see Fig. 1. The gap width, $d$, is nominally set at $0.5 \mathrm{~cm}$. Electromagnetic power is driven radially inward to the simulation region at $r=11 \mathrm{~cm}$ and the simulation is terminated by a fixed impedance load at $z=1 \mathrm{~cm}$. The nominal operating parameters at the load are $V_{\text {Load }}=$ $3 \mathrm{MV}$ and $I_{\text {Load }}=20 \mathrm{MA}$. The parallel electrode region extending from $r=7 \mathrm{~cm}$ to $r=4 \mathrm{~cm}$ (or $r=2 \mathrm{~cm}$ for some cases) is the primary simulation region where comparisons to the "high enhancement" ion loss current model of Ref. [5] are made. SCL emission of ions $\left(\mathrm{H}^{+}\right.$and/ or $\mathrm{O}^{+}$) occur along the anode in this region. An electron sheath is injected at $r=11 \mathrm{~cm}$ with a nominal current of $\sim 500 \mathrm{kA}$. This sheath is injected near the anode, similar to the high-density portion of the electron sheath entering the inner MITL in 3D convolute simulations [2]. As the sheath propagates radially, vortices rapidly form which thicken the sheath resulting in an average density profile in the ion emission region that fills the AK gap. See Appendix A for more details about the injected electron sheath characteristics and dynamics.

The simulations are fully kinetic, fully EM, relativistic and use an explicit field solver. The spatial resolution in the

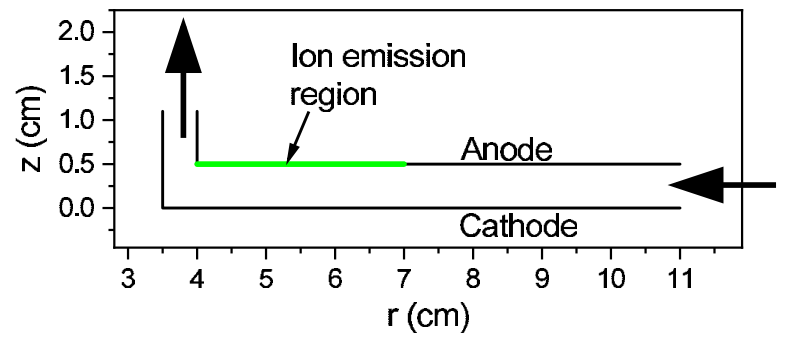

FIG. 1. Schematic of the 2D simulation geometry. Electromagnetic power and the electron sheath are injected radially at the $r=11 \mathrm{~cm}$ inlet boundary. The transmission line is terminated at $z=1 \mathrm{~cm}$ by a fixed impedance load. 
radial direction is $50 \mu \mathrm{m}$ and in the axial direction (across the AK gap) it is $12.5 \mu \mathrm{m}$. An electron sheath is injected at $r=11 \mathrm{~cm}$ into the MITL gap. In most cases, the sheath is injected near the anode with a uniform current density, a drift energy of $\sim 3 \mathrm{keV}$ in the $-r$ direction, and a temperature of $1 \mathrm{MeV}$. (See Appendix A for a discussion of the impact of the injected sheath temperature on the sheath current.) As the sheath propagates into the MITL, vortices are rapidly formed. When the sheath enters the ion emission region at $r=7 \mathrm{~cm}$, the sheath is strongly disrupted and rapidly fills the AK gap. The sheath properties in this region are discussed in more detail in Sec. III. The properties of the injected electron sheath are discussed further in Appendix A.

In addition to the injected electron sheath, electrons are emitted from cathode surfaces in the ion emission region, $4 \mathrm{~cm} \leq r \leq 7 \mathrm{~cm}$, using a relativistically correct SCL model [14] with an electric field threshold of $240 \mathrm{kV} / \mathrm{cm}$. This emission threshold is consistent with published estimates for stainless steel and aluminum cathode materials (see, for example, Ref. [15]). Overall we find that electrons emitted from the cathode do not play a significant role in the simulation results presented in this work. Tests with additional electron emission upstream of the ion emission resulted in a well-insulated electron sheath formed upstream of the ion emission region which did not significantly affect the simulation results presented here.

\section{BASELINE SIMULATION RESULT}

In the "high enhancement" limit for ion losses, described in Ref. [5], the charge within the sheath that spans the AK gap is large enough to produce a virtual cathode, resulting in ion current densities crossing the AK gap that can greatly exceed the conventional CL limit (in CGS units),

$$
j_{\mathrm{CL}}=\left(\frac{2 e Z}{m_{i}}\right)^{1 / 2} \frac{V^{3 / 2}}{9 \pi d^{2}},
$$

where $e Z$ is the ion charge, $m_{i}$ is the ion mass, $V$ is the voltage and $d$ is the AK gap width. For our baseline simulation parameters $\left(V=3 \mathrm{MV}, Z=1, m_{i}=16 m_{p}\right.$ where $m_{p}$ is the proton mass, and $d=0.5 \mathrm{~cm}), j_{\mathrm{CL}}$ is approximately $283 \mathrm{~A} / \mathrm{cm}^{2}$. Since the ion mass to charge ratio is sufficiently large to prevent significant magnetic deflection (or insulation) of the $\mathrm{O}^{+1}$ ions at $20 \mathrm{MA}$, the $\mathrm{CL}$ ion loss current for our system is roughly $I=j_{\mathrm{CL}}$ Area $=j_{\mathrm{CL}} \pi\left(7^{2}-4^{2}\right)=29.3 \mathrm{kA}$.

The injected electron sheath current is approximately 0.5 MA. The dynamics of the electron sheath as it propagates along the constant gap, variable vacuum impedance radial line is described in Appendix A. At the selected location for ion emission, $r=7 \mathrm{~cm}$, the electron sheath expands to fill the gap between the electrodes. Although the sheath upstream of, and within, the ion emission region is dynamic, with electron vortex formation resulting in ion

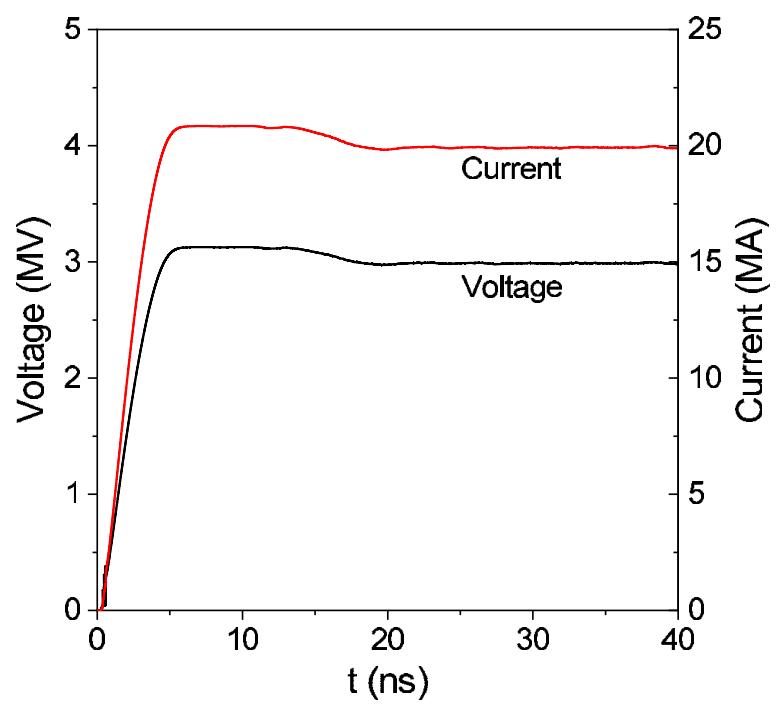

FIG. 2. Baseline simulation voltage and current illustrating the equilibrium values established between 20 and $40 \mathrm{~ns}$.

density variations along the emission region, the time and spatial average of the ion and electron densities provides quasi-equilibrium values for direct comparison to the WDC-HE model. The ion loss current obtained in the baseline simulation reported here is $\sim 800 \mathrm{kA}$, giving an average enhancement factor $\langle\eta\rangle \simeq 27$.

The system voltage and current as a function of time are shown in Fig. 2. All simulation quantities used for comparison with the model of Ref. [5] are temporally averaged between 30 and $40 \mathrm{~ns}$. The electron and ion number densities at times 30.1, 30.2, 30.3, and 30.4 ns are plotted in Fig. 3. These snap shots illustrate the dynamics of the electron
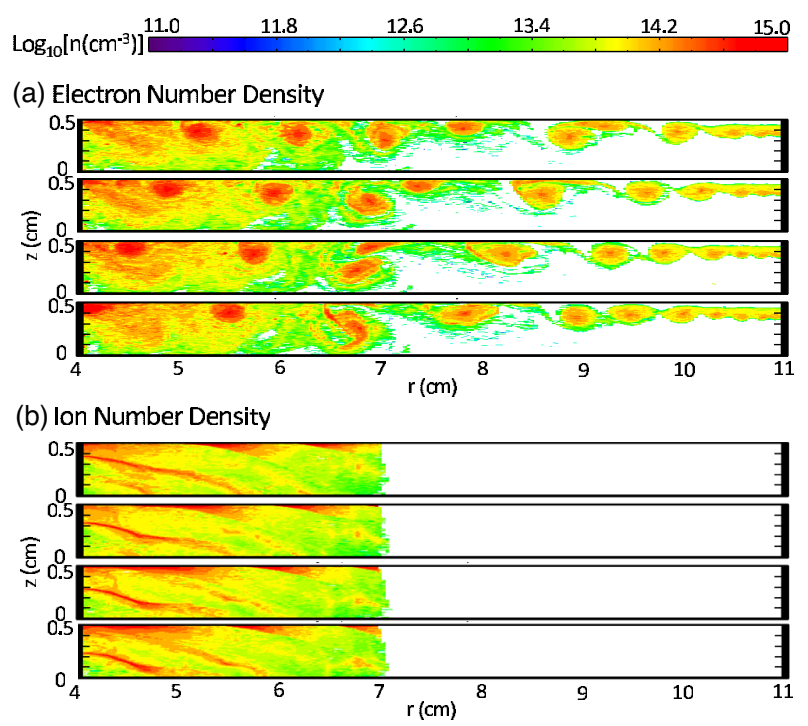

FIG. 3. (a) Electron and (b) ion $\left(\mathrm{O}^{+1}\right)$ number densities plotted at $\mathrm{t}=30.1,30.2,30.3$, and $30.4 \mathrm{~ns}$ intervals. The electron sheath is injected from the $r=11 \mathrm{~cm}$ boundary and $\mathrm{O}^{+1}$ ion emission is enabled along the anode between $r=4$ and $7 \mathrm{~cm}$. 
sheath, injected at $r=11 \mathrm{~cm}$, with vortices that rapidly form as the injected electron sheath propagates radially inward toward the load. The vortices result in variable charge and current densities in the electron sheath that, in turn, drive oscillations in the ion current density. To obtain quasiequilibrium values for the quantities used for comparison with the theoretical model, we temporally average data between 30 and $40 \mathrm{~ns}$ (in $0.1 \mathrm{~ns}$ steps). This timescale encompasses many vortex transit times through the simulation space and multiple ion sheath oscillation times.

The temporally averaged electron and ion densities at $r=5 \mathrm{~cm}$ are shown in Fig. 4(a). As used in the theoretical model of Ref. [5], the electron density shape is qualitatively a two-level profile with a high-density layer near the anode and a lower density layer filling the remainder of the AK gap. The ion density between the cathode and the high-density portion of the electron sheath is roughly $1 \times 10^{14} \mathrm{~cm}^{-3}$, and the lowdensity portion of electron sheath provides significant, although not complete, charge neutralization.

The $E_{z}$ and $B_{\theta}$ fields across the AK gap at $r=5 \mathrm{~cm}$ are shown in Fig. 4(b). Again, these are temporally and spatially averaged values as discussed above. The electric field approaches zero at $z \sim 0.2 \mathrm{~cm}$, indicating the presence of a virtual cathode as required by the theoretical model for enhanced ion current loss above the CL limit [5]. The electric field approaches $-17 \mathrm{MV} / \mathrm{cm}$ near the anode and rapidly returns to zero at the anode.
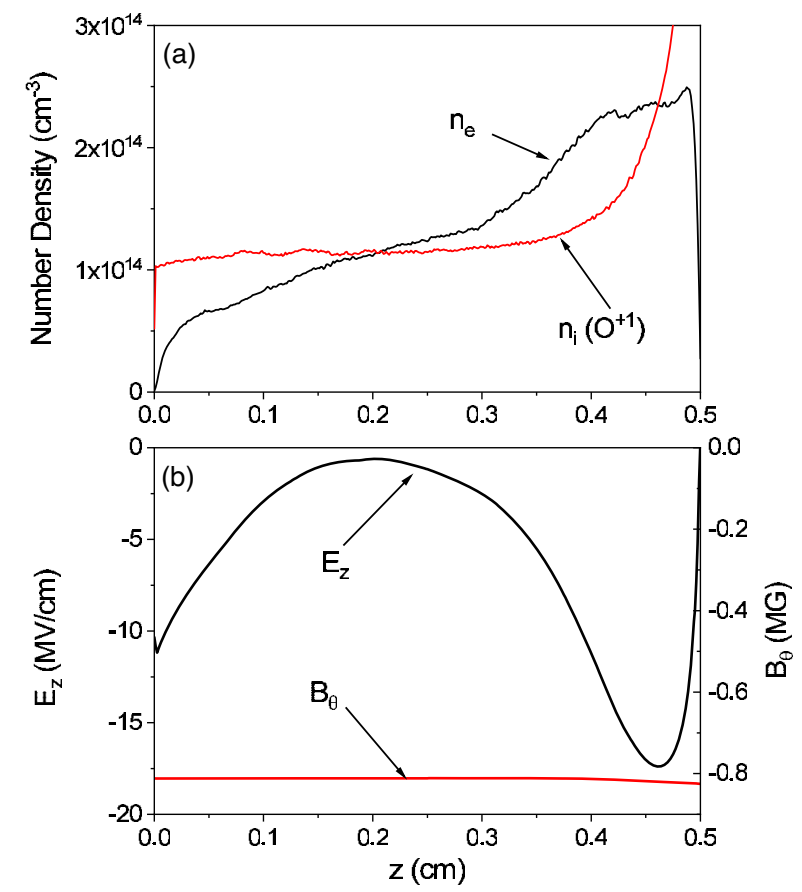

FIG. 4. Time averaged electron (black line) and ion (red line) number densities at $r=5 \mathrm{~cm}$ (spatially averaged between 4.9 and $5.1 \mathrm{~cm}$ ). Time averaged $E_{z}$ (black line) and $B_{\theta}$ (red line) fields at $r=5 \mathrm{~cm}$ (spatially averaged between 4.9 and $5.1 \mathrm{~cm}$ ). The cathode is at $z=0$ and the anode is at $z=0.5 \mathrm{~cm}$.

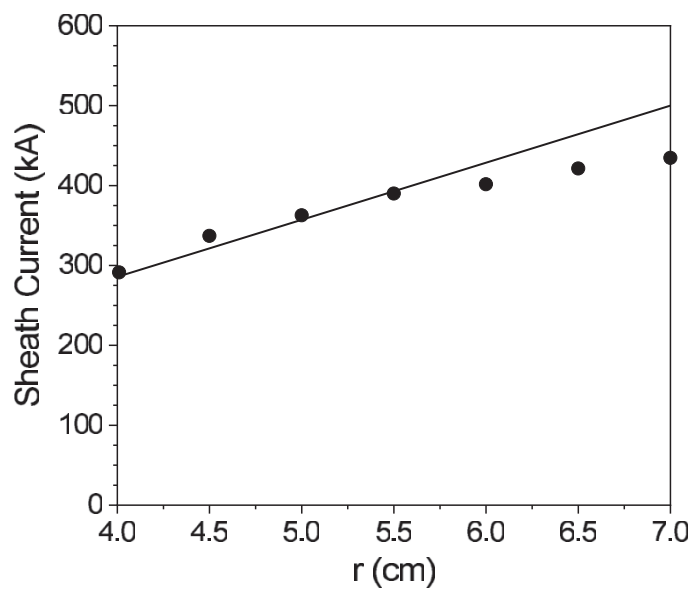

FIG. 5. Black data points indicate the temporally averaged electron sheath current as a function of radius from the baseline case. The solid line is the approximate sheath current as a function of radius from Eq. (5b) with $I_{e 0}=500 \mathrm{kA}$ and $r_{2}=7 \mathrm{~cm}$.

As the sheath propagates from $r=11 \mathrm{~cm}$ toward the load, the sheath dynamics result in losses of electron current to the anode as a function of radius. The sheath current as a function of radius is plotted in Fig. 5 (black data points). At $r=5 \mathrm{~cm}$, Fig. 5 indicates that the electron sheath current is roughly $350 \mathrm{kA}$ for this case. The sheath current as a function of radius can be roughly characterized as decreasing linearly with radius for the simulations presented here. The average electron current lost to the anode and the ion current lost to the cathode are plotted in Fig. 6. Again these curves can be roughly approximated as linear functions in the ion emission region, $r=4$ to $r=7 \mathrm{~cm}$.

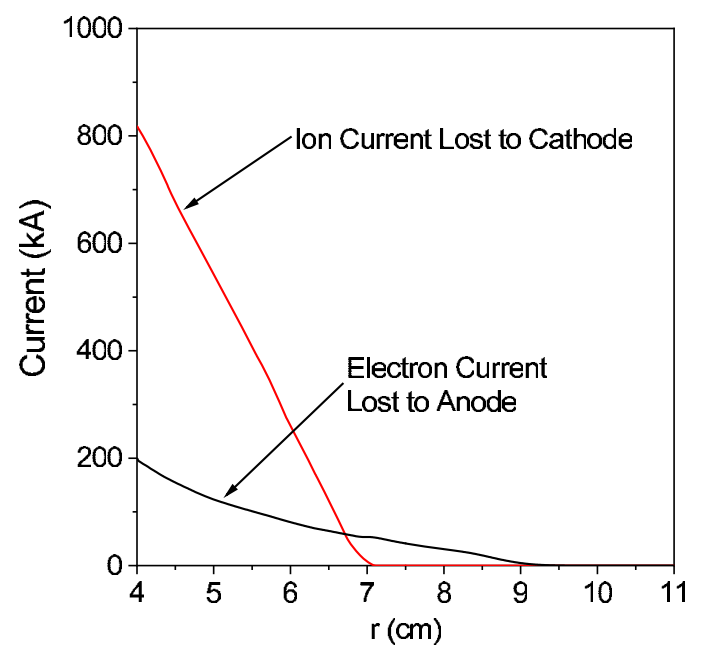

FIG. 6. Average electron current lost to the anode (black) and ion current lost to the cathode (red) as a function of radius from the baseline simulation. 
From Ref. [5], the enhancement factor is calculated as

$$
\eta(r)=\frac{9}{16} \gamma_{0}^{2}
$$

where

$$
\gamma_{0}^{2}=\left(B_{a}^{2}-B_{c}^{2}\right) \frac{d^{2}}{V^{2}}
$$

with $B_{a}$ and $B_{c}$ representing the azimuthal magnetic field at the anode and cathode, respectively. Using $B_{a}=2 \times 10^{7} /(5 r)$,

$$
\gamma_{0}^{2} \simeq 2\left(\frac{d}{5 V}\right)^{2} \frac{I_{a} I_{e}(r)}{r^{2}}
$$

where $I_{e}$ is the electron sheath current as a function of radius. For $V=3 \mathrm{MV}, I_{a}=20 \mathrm{MA}$, and $d=5 \mathrm{~mm}$, this expression can be integrated between $r=4 \mathrm{~cm}$ and $7 \mathrm{~cm}$ and gives an average ion loss current enhancement of $\langle\eta\rangle=7.63 \times 10^{-5} I_{e}$. For $I_{e} \simeq 350 \mathrm{kA}$ (see Fig. 5), $\langle\eta\rangle=26.7$, which is in very good agreement with the simulation results.

\section{SINGLE ION SPECIES PARAMETER VARIATIONS}

The baseline case described in Sec. III was modified to explore a number of parameter variations. The results of these simulations are given in Table I. The physical AK gap, operating voltage and current are listed along with injected electron sheath current. The emitted ion species and average enhancement to the ion current loss, $\eta$, is also given. Finally the simulated total ion loss current is listed along with the value calculated from the WDC-HE model [5],

$$
\begin{gathered}
I_{\mathrm{loss}}[\mathrm{MA}] \simeq \frac{1.38 \times 10^{-3}}{\sqrt{\mu}}\left(\frac{I_{e 0} I_{a}}{\sqrt{V}}\right) \frac{r_{2}-r_{1}}{r_{2}}, \\
I_{e}(r) \simeq r I_{e 0} / r_{2},
\end{gathered}
$$

where $Z$ is the ion charge state $(Z=1$ for all cases presented here), $\mu=m / m_{p}$ is the ion mass relative to the mass of the proton, $I_{e 0}$ is the electron sheath current at $r=11 \mathrm{~cm}, I_{a}$ is the anode current, $V$ is the voltage, and $r_{1}$ and $r_{2}$ are the downstream and upstream limits of the ion emission region, respectively. We find that the sheath current decreases linearly with radius in our simulations according to Eq. (5b).

For the range of parameters given in Table I, the overall agreement between the simulated ion loss currents and the WDC-HE model given in Eq. (5a) is very good. Besides the baseline (case 0), cases 2, 3, 5, and 6 all use the same voltage and current, and all give good agreement with the WDC-HE model. Case 5 is particularly notable as the simulation geometry was modified to include sheath propagation and ion emission down to $r=2 \mathrm{~cm}$. Case 6 uses a smaller AK gap, and the ion current losses are essentially unchanged, confirming the independence from AK gap given by the WDC-HE model. Case 7, at 2.7 MV and 9.0 MA, uses $\mathrm{H}^{+}$and scales as expected (see case 3) with ion species.

However, two cases have simulated ion loss currents that do not agree as well with the WDC-HE model (but are still within $\sim 20 \%$ of the model). Case 1, with an injected electron sheath current of $1.0 \mathrm{MA}$, shows a relatively large disagreement with the WDC-HE model. This is due, in part, to the large sheath density driving an electric field reversal in the AK gap in the ion emission region. This field structure modifies the ion current density that is lost to the cathode in a way that is not included in the WDC-HE model.

In contrast case 4 , operated at $\sim 0.95 \mathrm{MV}$, gives a lower than predicted ion current loss, relative to the WDC-HE model. Once again, a reversal in the electric field, driven by a reversal in electron sheath current in the ion emission region, is a condition not included in the WDC-HE model.

Overall, the cases shown in Table I exhibit relatively large ion current losses, well over the CL limit, for a wide range of realizable $Z$ operating parameters. Although the electron density distribution is not precisely the same as utilized in Ref. [5] the enhancement factors observed in our

\begin{tabular}{|c|c|c|c|c|c|c|c|c|c|}
\hline Case & $\begin{array}{c}\text { Gap } \\
(\mathrm{mm})\end{array}$ & $\begin{array}{l}\text { Voltage } \\
(\mathrm{MV})\end{array}$ & $\begin{array}{l}\text { Current } \\
\text { (MA) }\end{array}$ & $\begin{array}{c}\text { Injected } \\
\text { Current (MA) }\end{array}$ & $\begin{array}{c}\text { Ion } \\
\text { Species }\end{array}$ & $\begin{array}{c}\text { Radial } \\
\text { Extent }(\mathrm{cm})\end{array}$ & Average $\eta$ & $\begin{array}{c}\text { Ion Loss Current } \\
\text { (MA) }\end{array}$ & $\begin{array}{c}\text { WDC-HE } \\
\text { Theory } \\
\text { (MA) }\end{array}$ \\
\hline 0 & 5.0 & 3.0 & 20.0 & 0.5 & $\mathrm{O}^{+}$ & $4-7$ & 27.4 & 0.82 & 0.85 \\
\hline 1 & 5.0 & 3.0 & 20.0 & 1.0 & $\mathrm{O}^{+}$ & $4-7$ & 69.8 & 2.2 & 1.71 \\
\hline 2 & 5.0 & 3.0 & 20.0 & 0.25 & $\mathrm{O}^{+}$ & $4-7$ & 14.7 & 0.5 & 0.43 \\
\hline 3 & 5.0 & 3.0 & 10.0 & 0.5 & $\mathrm{O}^{+}$ & $4-7$ & 15.5 & 0.5 & 0.43 \\
\hline 4 & 5.0 & 0.95 & 19.0 & 0.5 & $\mathrm{O}^{+}$ & $4-7$ & 19.7 & 1.0 & 1.4 \\
\hline 5 & 5.0 & 3.0 & 20.0 & 0.5 & $\mathrm{O}^{+}$ & $2-7$ & 34.6 & 1.3 & 1.4 \\
\hline 6 & 2.5 & 3.0 & 20.0 & 0.5 & $\mathrm{O}^{+}$ & $4-7$ & 8.1 & 0.95 & 0.85 \\
\hline 7 & 5.0 & 2.7 & 9.0 & 0.5 & $\mathrm{H}^{+}$ & $4-7$ & 13.9 & 1.4 & 1.6 \\
\hline
\end{tabular}
single ion species simulations are remarkably close to those found in the HE case of that reference. To elucidate how this agreement comes about, we start as in Ref. [5], with

TABLE I. Summary of the single ion species, 2D EM simulation results. 
Poisson's and Ampere's equations in planar steady state, assuming laminar $E \times B$ electron drift, and negligible positive ion deflection:

$$
\begin{gathered}
\phi^{\prime \prime}(\nu)=\frac{\alpha}{\sqrt{1-\phi(\nu)}}-\beta(\nu), \\
\gamma_{0}^{2}=b_{a}^{2}-b_{c}^{2}=2 \int_{0}^{1} \beta(\nu) \phi^{\prime}(\nu) d \nu .
\end{gathered}
$$

Equations (6) are Eqs. (8), (9a), and (9b) of Ref. [5] expressed in dimensionless variables; $\phi=\phi / V, \nu=z / d$, $b=B d / V$, and $\beta=4 \pi e n_{e} d^{2} / V$. We integrate Eq. (6a) after multiplying left and right sides by $\phi^{\prime}$, and employing (6b), obtaining:

$$
\alpha=\left[\gamma_{0}^{2}(0)-\phi^{\prime \prime}\left(0^{+}\right)\right] / 4
$$

where $9 \alpha / 4$ is the ratio of the enhanced ion current density and the $\mathrm{CL}$ value in the absence of electron charge. By $0^{+}$ we mean a point just above the locally emitted electron sheath, which provides charge but carries a negligible amount of current. Recalling from Ref. [5] that the enhancement factor $\eta=9 \alpha / 4$, we obtain;

$$
\eta=\frac{9}{16}\left(\gamma_{0}^{2}-\left[\phi^{\prime}\left(0^{+}\right)\right]^{2}\right)
$$

Equation (8) is a significant result: given an electron current determining the magnitude of the diamagnetic effect, represented in dimensionless units by $\gamma_{0}^{2}$, any electron charge distribution, given in dimensionless units by $\beta(\nu)$, satisfies Eq. (8), as long as the electron current sheath undergoes $E \times B$ flow, determining the electron velocity contributing to the electron current sheath.

Our present simulations demonstrate that if such electron sheath, however it is created, is injected into an ion emitting region and "survives" (that is, it is not entirely lost to the electrodes), its current determines the enhancement factor. Moreover, if that current is sufficiently high, the ion loss it produces is very close to the $\mathrm{HE}$ regime [5], given that $\gamma_{0}^{2} \geq \pi^{2}$ and $\gamma_{0}^{2} \gg\left[\phi^{\prime}\left(0^{+}\right)\right]^{2}$. The first is the condition that the "true virtual cathode" is inside the physical gap, and the second assures that the HE result, obtained when $\phi^{\prime}\left(0^{+}\right)=0$, and that of Eq. (8) are indeed close. Typically, in all cases simulated in this work with one heavy ion species, this is indeed the case, and we observe enhancement factors within $10 \%$ to $20 \%$ of the $\mathrm{HE}$ regime for the given value of the "surviving" electron sheath current at that given point.

\section{1D MULTIPLE ION SPECIES LOSS CURRENT SIMULATIONS}

As a simple test of the WDC-HE model for the case of two ion species, a series of 1D electrostatic (ES) simulations were carried out using an immobile electron density profile that enables ion currents in the HE limit. Optionally, an externally applied magnetic field, transverse to the gap, is included. The spatial resolution is $\Delta z=12.5 \mu \mathrm{m}$. The simulation results and comparisons to the WDC-HE model are summarized in Table II. For cases shown in Table II, $V=3 \mathrm{MV}, r=5 \mathrm{~cm}$, $d=2 \mathrm{~mm}, I_{e}=0.5 \mathrm{MA}$, and $I_{a}=20 \mathrm{MA}$. For reference, the $\mathrm{CL}$ ion current densities for $\mathrm{H}^{+}$and $\mathrm{O}^{+}$are $7.1 \mathrm{kA} / \mathrm{cm}^{2}$ and $1.8 \mathrm{kA} / \mathrm{cm}^{2}$, respectively. An immobile electron density profile is set according to

$$
n_{e}(z)= \begin{cases}1.310 \times 10^{14} \mathrm{~cm}^{-3}, & 0 \leq z \leq a_{1} d \\ 2.619 \times 10^{14} \mathrm{~cm}^{-3}, & a_{1} d<z \leq d\end{cases}
$$

where $a_{1}=0.11635, z$ is the position across the gap with the cathode at $z=0$, and the anode at $z=d$. These values are obtained for the WDC-HE limit by noting that $\beta_{2}=\gamma_{0}^{2} / 4$ with $\beta_{2}=4 \pi e n_{e 2} d^{2} / V$ (see Sec. IIB of Ref. [5] for more details.) Ions are emitted from the anode using the CHICAGO CL emission algorithm [14], modified to randomly emit one or two ion species with a prescribed probability. After the simulation has reached an equilibrium, the ion current densities lost to the anode are obtained and listed in Table II in the columns labeled $j_{\mathrm{O}}$ and $j_{\mathrm{H}}$ representing the oxygen and hydrogen ion current densities,

\begin{tabular}{|c|c|c|c|c|c|c|c|}
\hline$B(\mathrm{MG})$ & $\mathrm{O}^{+}$fraction & $\mathrm{H}^{+}$fraction & $\sqrt{1 / \mu}$ & $j_{\mathrm{O}}\left(\mathrm{kA} / \mathrm{cm}^{2}\right)$ & $j_{\mathrm{H}}\left(\mathrm{kA} / \mathrm{cm}^{2}\right)$ & $j_{\text {tot }}\left(\mathrm{kA} / \mathrm{cm}^{2}\right)$ & WDC-HE NMI $\left(\mathrm{kA} / \mathrm{cm}^{2}\right)$ \\
\hline 0 & 0 & 1.0 & 1.0 & 0.0 & 50.3 & 50.3 & 50.8 \\
\hline 0.8 & 0 & 1.0 & 1.0 & 0.0 & 48.3 & 48.3 & 50.8 \\
\hline 0 & 1.0 & 0.0 & 0.25 & 12.6 & 0.0 & 12.6 & 12.7 \\
\hline 0.8 & 1.0 & 0.0 & 0.25 & 12.6 & 0.0 & 12.6 & 12.7 \\
\hline 0 & $1 / 3$ & $2 / 3$ & 0.75 & 4.1 & 33.6 & 37.7 & 38.1 \\
\hline 0.8 & $1 / 3$ & $2 / 3$ & 0.75 & 4.1 & 32.5 & 36.6 & 38.1 \\
\hline 0 & $1 / 2$ & $1 / 2$ & 0.625 & 6.2 & 25.4 & 31.6 & 31.8 \\
\hline 0.8 & $1 / 2$ & $1 / 2$ & 0.625 & 6.2 & 23.3 & 29.5 & 31.8 \\
\hline
\end{tabular}
respectively. The sum of these two ion current densities is listed as the total ion current density lost to the cathode, $j_{\text {tot }}$.

TABLE II. Summary of the single and double ion species, 1D ES simulation results and comparisons with the WDC-HE model. For all cases, $V=3 \mathrm{MV}, r=5 \mathrm{~cm}, d=2 \mathrm{~mm}, I_{e}=0.5 \mathrm{MA}$, and $I_{a}=20 \mathrm{MA}$. 
For the cases with two ion species shown in Table II, the summation rule from Eq. (12) is used. The values of $\sqrt{1 / \mu}$ are listed in Table II. The total ion current density lost to the anode in the HE limit at a radius $r$ can be written as

$$
j_{\text {loss }}\left[\frac{\mathrm{A}}{\mathrm{cm}^{2}}\right] \simeq \frac{2.2 \times 10^{-4}}{\sqrt{\mu}}\left(\frac{I_{e 0} I_{a}}{r^{2} \sqrt{V}}\right) .
$$

For each case listed in Table II, the result of Eq. (10) is listed in the column labeled WDC-HE NMI, since Eq. (10) does not include the effects of the applied magnetic field present in some of the simulations. For most all of the $B=0$ cases, the simulation results are in very good agreement with $j_{\text {loss. }}$. The inclusion of the magnetic field reduces the $j_{\mathrm{H}}$ somewhat (for cases that include $\mathrm{H}^{+}$ion emission), but overall the high current losses obtained in the HE limit are retained throughout.

\section{TWO ION SPECIES CASE STUDY}

Initial 2D EM CHICAGO simulations showed that the partial insulation of $\mathrm{H}^{+}$ions tended to reduce the $\mathrm{O}^{+}$ion current losses. The complexity of the electron sheath dynamics in the ion emission region made analysis of the ion losses difficult to understand. To address this, two 2D EM simulations were set up that used an immobile electron sheath with a prescribed two-level (in z) density profile that varied in $r$ to remove the complication of the electron sheath dynamics from the problem. These simulations were run to quasiequilibrium with $V=3 \mathrm{MV}$, $I_{a}=20 \mathrm{MA}$, and $I_{e}=5 \times 10^{5} \mathrm{r}(\mathrm{cm}) / 7 \mathrm{~A}$. The ions were emitted with the ratio $\mathrm{H}^{+} / \mathrm{O}^{+}$of $0.666 / 0.333$. The $\mathrm{AK}$ gaps were set to $d=5 \mathrm{~mm}$ and $2.5 \mathrm{~mm}$. The (immobile) electron sheath number density was set with the following functional form

$n_{e}(r, z)=\left\{\begin{array}{lll}b_{1} / r, & \text { for } 2 \leq r \leq 7, & g(r)<z \leq 1, \\ b_{2} / r, & \text { for } 2 \leq r \leq 7, & 0 \leq z \leq g(r),\end{array}\right.$

for $g(r)=d\left(1-a_{2} \sqrt{r}\right)$, with $d$ and $r$ in $\mathrm{cm}$ and $b_{1}=9.475 \times 10^{14}, b_{2}=4.737 \times 10^{14}$, and $a_{2}=0.09293$. This electron density corresponds to the WDC-HE limit of Ref. [5] and assumes the linear radial dependence of the electron sheath current given by Eq. (5b).

The ion number density for $\mathrm{H}^{+}$and $\mathrm{O}^{+}$are shown in the upper frames of Fig. 7 for the 3-mm gap case to illustrate the NMI and MI regions along the radial MITL. We define the radial position along the transmission line where the $\mathrm{H}^{+}$ Larmor radius, $\lambda_{L}$ is equal to the AK gap $d$ to be $r_{L}$. For radii greater than $r_{L} \simeq 4.7 \mathrm{~cm}$, where the $\mathrm{H}^{+}$Larmor radius $\lambda_{L} \gtrsim d$, the $\mathrm{H}^{+}$and $\mathrm{O}^{+}$ions are NMI. For radii less than $r_{L}$, where $\lambda_{L} \lesssim d$, the $\mathrm{H}^{+}$ions transition from NMI to PMI to fully MI with decreasing radius, resulting in the formation

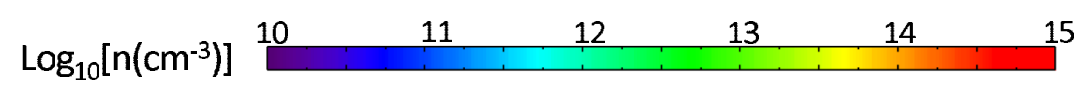

(a) $\mathrm{H}^{+}$

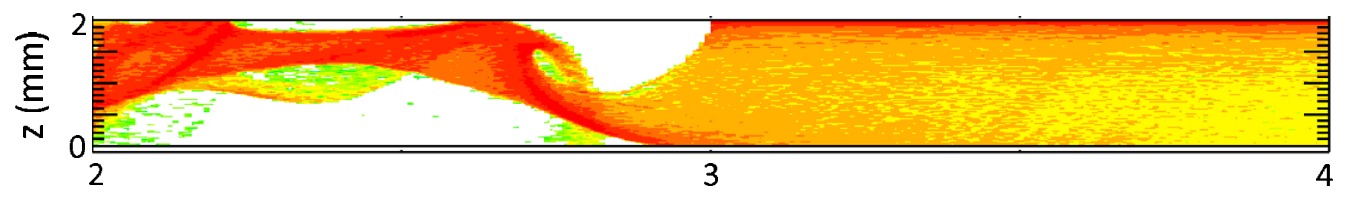

(b) $\mathrm{O}^{+}$
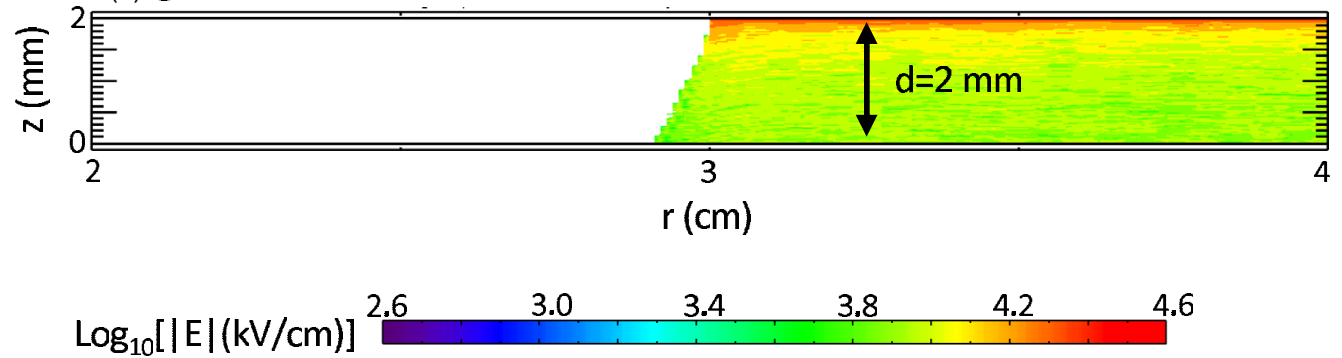

(c) $|\mathbf{E}|$

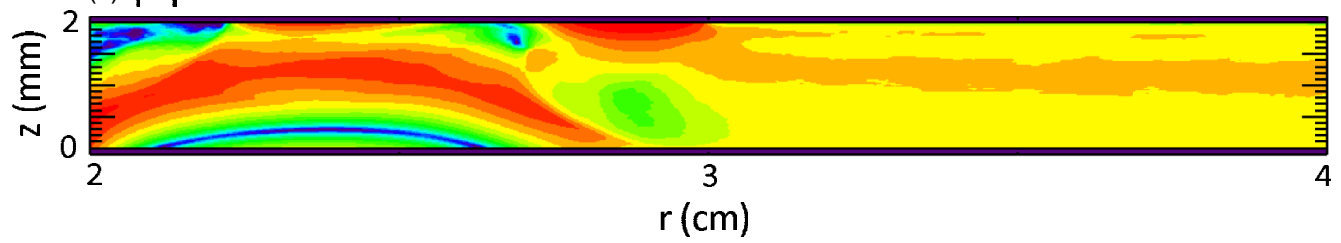

FIG. 7. Snap shots of (a) the $\mathrm{H}^{+}$number density, (b) the $\mathrm{O}^{+}$number density, and (c) the electric field magnitude for the immobile electron, two-ion-species case with a 3-mm AK gap. 
TABLE III. Summary of the two ion species, 2D EM simulation results. All cases use an $\mathrm{H}^{+}$fraction of $2 / 3$, giving $\sqrt{1 / \Lambda}=0.75$, and an ion emission region extending between $r=3 \mathrm{~cm}$ and $7 \mathrm{~cm}$.

\begin{tabular}{|c|c|c|c|c|c|c|c|}
\hline Description & $\begin{array}{l}\text { Gap } \\
(\mathrm{mm})\end{array}$ & $\begin{array}{c}I_{\text {sheath }} \text { at } \\
r=5 \mathrm{~cm} \\
(\mathrm{kA})\end{array}$ & $\begin{array}{c}\text { Radius } \\
r_{L} \text { for } d=\lambda_{L} \\
\text { for } \mathrm{H}^{+}(\mathrm{cm})\end{array}$ & $I_{\mathrm{O}}(\mathrm{MA})$ & $I_{\mathrm{H}}(\mathrm{MA})$ & $I_{\text {tot }}(\mathrm{MA})$ & $\begin{array}{c}\text { WDC-HE NMI } \\
\text { Theory (MA) }\end{array}$ \\
\hline $\begin{array}{l}\text { Immobile electrons } 3.07 \mathrm{MV} \text {, } \\
20.5 \mathrm{MA}, \mathrm{MI}\end{array}$ & 5 & ... & 8.1 & 0.06 & 0.15 & 0.21 & 0 \\
\hline $\begin{array}{l}\text { Immobile electrons } 2.89 \mathrm{MV} \text {, } \\
19.3 \mathrm{MA}, \mathrm{PMI}\end{array}$ & 3 & $\cdots$ & 4.7 & 0.17 & 1.15 & 1.32 & 1.85 \\
\hline Immobile electrons $2.83 \mathrm{MV}, 18.9 \mathrm{MA}, \mathrm{NMI}$ & 2 & $\ldots$ & 3.1 & 0.23 & 1.79 & 2.02 & 3.16 \\
\hline Dynamic electrons $3.03 \mathrm{MV}, 20.2 \mathrm{MA}$, MI & 5 & 340 & 8.0 & 0.12 & 0.31 & 0.43 & 0 \\
\hline Dynamic electrons $3.03 \mathrm{MV}, 20.2 \mathrm{MA}$, PMI & 3 & 360 & 4.8 & 0.26 & 1.72 & 1.92 & 1.87 \\
\hline Dynamic electrons $2.47 \mathrm{MV}, 19.8 \mathrm{MA}$, NMI & 2 & 360 & 3.2 & 0.47 & 3.57 & 4.04 & 3.23 \\
\hline
\end{tabular}

of an $\mathrm{H}^{+}$ion sheath that turns off or screens the SCL emission of $\mathrm{O}^{+}$ions for radii less than $\sim 3.8 \mathrm{~cm}$. The strong reduction in the electric field magnitude along the anode can be seen in frame (c) for radii less than $\sim 3.8 \mathrm{~cm}$. This case is listed as PMI in Table III because a portion of the ion emission region is in the NMI limit and a portion is in the NMI limit, i.e., $\lambda_{L}>d$.

Table III summarizes the results of these simulations. After a brief description of the simulation case and the physical AK gap used, the third column gives the radius at which $\lambda_{L}$ for $\mathrm{H}^{+}$equals the $\mathrm{AK}$ gap. The $\mathrm{H}^{+}$fraction is given in the fourth column and the fifth column gives the value of $\sqrt{1 / \Lambda} \equiv \sqrt{Z / \mu}$ from the summation rule,

$$
\frac{1}{\sqrt{\Lambda}}=\frac{k_{1}}{\sqrt{\Lambda_{1}}}+\frac{k_{2}}{\sqrt{\Lambda_{2}}}
$$

where $k_{i}$ is the concentration percentage of the $i$ th ion species of charge state $Z_{i}, \Lambda_{i}=\mu_{i} / Z_{i}, \mu_{i}=m_{i} / m_{p}$ is the ion mass relative to the mass of the proton, and $\Lambda$ is the equivalent ion reduced mass/charge ratio of ion mix considered. Equation (12) is a generalized form of the mass summation rule of Ref. [16] for arbitrary mix of positive ion species, see Appendix C. The next three columns give the ion current lost to the cathode for the individual ion species and the sum of these two values. The final column gives the NMI ion current lost to the anode from the WDC-HE model starting from Eq. (5) described below starting with Eq. (13).

The final column in Table III provides the approximation given by Eq. (13), where we replace $r_{1}$ by $r_{L}$ with the stipulation that if $r_{L} \geq r_{2}$ the loss current vanishes. That is,

$$
I_{\mathrm{loss}}[\mathrm{MA}] \simeq \begin{cases}\simeq 1.38 \times 10^{-3}(0.75)\left(I_{e r_{2}} I_{a} / \sqrt{V}\right)\left(r_{2}-r_{L}\right) / r_{2}, & \text { for } r_{2}>r_{L} \\ 0, & \text { for } r_{2} \leq r_{L}\end{cases}
$$

We have also set $\sqrt{Z / \mu}=0.75$ which corresponds to Eq. (12) for $k_{1}=2 / 3, \quad k_{2}=1 / 3, \quad \mu_{1}=1$, and $\mu_{2}=16$; that is two hydrogen ions for each oxygen ion. Clearly the approximation for the WDC-HE case for the given ion mixture if the gap is less than the value of the hydrogen Larmor radius at that time and location, and zero otherwise.

We remark that the simple approximation of Eq. (13) agrees with the simulation results presented in Table III to within about $30 \%$. This is noteworthy because the approximation is based upon the 1D planar WDC $\mathrm{HE}$ regime, complemented by the simulation findings on the radial dependence of the injected electron sheath current and assumed radial cut-off value using the definition of the $\mathrm{H}^{+}$Larmor radius in vacuum, starting at rest at the anode.

\section{SUMMARY AND DISCUSSION}

Numerical simulations of the WDC-HE limit of Ref. [5] have been carried out to verify the model for a single, NMI ion species. The simulations have been executed in 1D ES to test the theoretical model in a direct manner and 2D EM to test the applicability of the model in a more realistic manner where electron sheath dynamics drive fluctuations in the EM fields and ion current densities. Despite the complexities of the sheath dynamics obtained in the $2 \mathrm{D}$ simulations, the overall agreement with the predictions of the theoretical model of Ref. [5] in the high enhancement regime are very compelling.

The most striking result of our present research is the finding that the presence of more than one ion species emitted from the anode plasma is paramount in ascertaining 
the importance of ion current losses in the convolute and inner MITL of $Z$, and similar pulsed power machines. In particular, the most important effect is the influence of lighter ion species. By lighter we mean ion species that could be potentially, at given radial positions and times in the pulse, be magnetically insulated. This is indeed the case for $\mathrm{H}^{+}$, but it could also be the case, for instance, of $\mathrm{C}^{+4}$. What our present analysis and simulation results imply is that, if the lighter ions are a significant fraction of the anode plasma composition and are magnetically insulated over the whole region of interest, then the ion losses become relatively small. On the other hand, if the cathode and anode plasmas expand such as to reduce the effective AK gap to be smaller than the lighter species Larmor radius, then the ion current losses can be sizable. In that situation, they do not provide shielding and dominate the ion current loss. This can be understood from the generalized summation rule given by Eq. (12) and derived in Appendix C; if $\mathrm{H}^{+}$is present and not MI, the effective loss is a significant fraction of what it would be if only $\mathrm{H}^{+}$is present and NMI. For instance, for the $\mathrm{H}^{+} 2 / 3, \mathrm{O}^{+} 1 / 3$ case, that fraction is 0.75 . These results, both the main finding of the role of lighter ions in the presence of more than one ion species, and the generalized summation rule derived in Appendix C, are to our knowledge, a new addition to the understanding of current losses on $Z$ and similar pulsed power machines.

The simulations presented here explicitly exhibit the dependence of ion current losses with respect to voltage, current, and AK gap as a function of time and location in the inner MITL. We do expect the presence of multiple ion species in the anode plasma, making the most relevant results those corresponding to Sec. VI. These dependencies are clearly expressed in Eqs. (10) and (12) if no species is $\mathrm{MI}$ (or if there is a single ion species that is NMI). If there is an ion species which, at a given time and position, magnetically insulated the ion current losses, at that time and position, are for all practical purposes negligible. Therefore, in order to complete the quantitative description of ion current losses in the inner MITL, corresponding to the physics employed in performing the present simulations, we need to know if the lightest ion species Larmor radius is larger or smaller than the effective AK gap, at a given time and position. In turn this implies the need to know electrode plasma expansion rates. We also need to know the magnitude of the electron current sheath. In this respect our simulations indicate that for a constant gap radial MITL, the electron sheath current decreases proportional to radius, thus providing a reasonable conjecture for the electron sheath survival rate in the inner MITL. Finally, we need to know what is, as a function of time, the electron sheath current magnitude and density profile entering the inner MITL from the convolute. We do have factor of two or better estimates of the sheath currents in the four outer MITLs just upstream of the convolute as a function of voltage, current, and effective gap. These estimates need to be refined by simulations and experimental measurements.
Assuming that in an actual experiment the losses approximately follow the scaling we find in steady state, that dependence could be used in future simulations, and more importantly in dedicated $Z$ experiments designed to ascertain inner MITL current losses and the main loss mechanism. In summarizing these findings, we remark again that we are still left with determining, either through experimental measurement or numerical simulations, electrode plasma expansion rates, current losses at the convolute prior to the electron sheath entering the inner MITL, and with good accuracy the electron sheath currents entering the convolute from the four outer MITLs. In time-dependent simulations presently underway, we will attempt to elucidate, based on simulations of actual $Z$ experiments, those quantities treated as parameters. However, going beyond fitting will require careful simulations revisiting those processes as well as new measurements on $Z$.

\section{ACKNOWLEDGMENTS}

The authors thank E. Watson, J. Gansz-Torres, T. Genoni, C. Mostrom, I. Paraschiv, and D. Voss at Voss Scientific for assistance with the simulations and data analysis. We acknowledge helpful discussions with $\mathrm{M}$. Gomez, M. Hess, C. Jennings, D. Lamppa, and A. Porwitzky at Sandia National Laboratories, and $\mathrm{K}$. LeChien and W. Stygar at Lawrence Livermore National Laboratory. This work was partially supported by LDRD Project No. 209240 at Sandia National Laboratories. Sandia National Laboratories is a multi-mission laboratory managed and operated by National Technology and Engineering Solutions of Sandia, LLC., a wholly owned subsidiary of Honeywell International, Inc., for the U.S. Department of Energy's National Nuclear Security Administration under Contract No. DE-NA0003525. This paper describes objective technical results and analysis. Any subjective views or opinions that might be expressed in the paper do not necessarily represent the views of the U.S. Department of Energy or the United States Government.

\section{APPENDIX A: ELECTRON SHEATH SIMULATION}

To illustrate the injected electron sheath properties and dynamics in the 2D EM simulations, a separate case was run without ion emission. The only source of charge particles in this case is the injected electron sheath. As discussed in Sec. III, the electron sheath is injected at $r=$ $11 \mathrm{~cm}$ between $z=3.5$ and $4.5 \mathrm{~mm}$ with a uniform density profile. The voltage and current of system is established in $\sim 10 \mathrm{~ns}$, and then the electron sheath is injected with a linearly rising ramp to the current density over $5 \mathrm{~ns}$, then is held constant at $150 \mathrm{kA} / \mathrm{cm}^{2}$. The electrons are given a drift momentum in the $-r$ direction of $0.1 \beta \gamma$ and a 


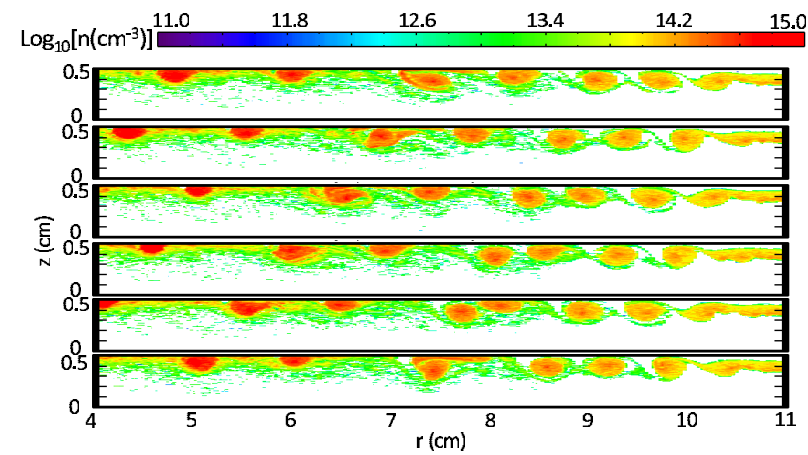

FIG. 8. Electron number density plotted at $39.0 \mathrm{~ns}$ (top) to $39.5 \mathrm{~ns}$ (bottom) in $0.1 \mathrm{~ns}$ intervals. The electron sheath is injected from the $r=11 \mathrm{~cm}$ boundary. This case does not include emission of ions from the anode.

temperature, $T_{e}$, of $1 \mathrm{MeV}$. This distribution provides a crude but reasonable approximation to the electron distribution function in the inner MITL as obtained in 3D convolute simulations $[2,17]$ driving a MagLIF load at $\sim 20$ MA load current and 2 MV. A smaller temperature for the injected electron sheath results in a somewhat larger sheath current that propagates forward, and vise versa. The azimuthal component of the electron velocity arises mainly from electron orbits that move radially inward and go around the anode posts before entering the inner MITL.

Figure 8 plots the electron sheath number density between $39.0 \mathrm{~ns}$ and $39.5 \mathrm{~ns}$ in $0.1 \mathrm{~ns}$ intervals. The sheath, injected with a uniform current and number density, rapidly develops vortex structures that propagate forward at an increasing velocity. Vortex formation is likely due to the stability of the injected electron stream (see, for example, Ref. [18]). Not all vortices are the same size or density, and therefore propagate at slightly different speeds. This allows vortices to overtake each other, and dissipate or even combine to form larger structures. Note that without the presence of the ions that are emitted for radii less than $7 \mathrm{~cm}$, the sheath does not fill the AK gap in the manner shown in Fig. 3. Although the sheath is broader, it remains much closer to the anode as expected from Ref. [5]. Further work is required to completely understand the dynamics of these vortices, the interaction with the ion region as shown in Fig. 3, and to understand the sheath properties emerging from the convolute in the coupled MITL-convolute system on $Z[1,2,17,19]$.

\section{APPENDIX B: SPATIAL RESOLUTION AND MACROPARTICLE NUMBERS}

Numerical parameters for the baseline case described in Sec. III were varied to test for convergence of the time and spatial averaged ion loss current density $j_{i}$ used as a key metric throughout this work. The spatial resolution was changed from the baseline case value of $(\delta r, \delta z)=$ $(50,12.5)$ (in $\mu \mathrm{m})$ to $(50,50)$ and $(100,100)$. In addition,

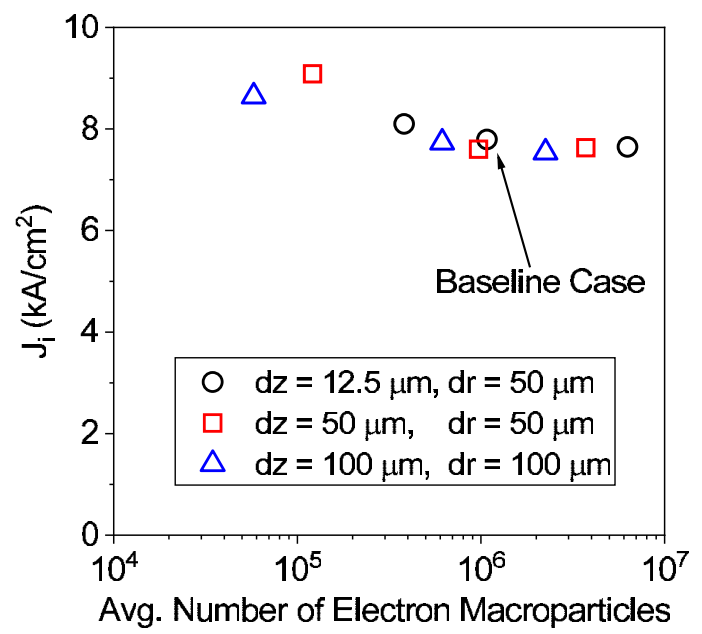

FIG. 9. Temporally and spatially averaged ion loss current density as a function of injected electron macroparticle number for different spatial resolutions. All results are for the baseline simulation case described in Sec. III.

the number of electron macroparticles injected at $r=$ $11 \mathrm{~cm}$ boundary were also varied for each of the spatial resolutions. The values of $j_{i}$ at $r=5 \mathrm{~cm}$ (temporally and spatially averaged as described in Sec. III) are shown in Fig. 9. Plotted as a function of the average number of injected electron macroparticles in the simulation, $j_{i}$ is relatively constant for electron macroparticle numbers $\gtrsim 10^{6}$ independent of spatial resolution.

We note that the $(100 \mu \mathrm{m}, 100 \mu \mathrm{m})$ resolution was selected for testing since 3D convolute simulations [2], which included a simple inner MITL section similar to the model used here, used approximately this spatial resolution.

\section{APPENDIX C: MULTIPLE ION SPECIES CL CURRENT DENSITY}

The multiple ion species current density formula presented in Ref. [16] is derived for single ion charge states only. Here we expand that derivation to include ion charges other than unity. We define $N$ to be the number of ion species in the anode plasma with normalized mass $\mu_{i}$, charge state $Z_{i}, \Lambda \equiv \mu_{i} / Z_{i}$, the fraction of the $i$ th species is $k_{i}$, with $n_{i}=k_{i} n$ and $\sum_{i=1}^{N} k_{i}=1$, where we denote by $n$ and $n_{i}$ the total and partial ion densities, respectively. We also define $j_{\mathrm{H}+}$ as the proton CL current density for a gap $d$ of potential $V$. Similarly, we define $j$ and $j_{i}$ as the total and partial current density, respectively. We then have the following summations;

$$
j=\sum_{i=1}^{N} j_{i},
$$

and 


$$
j_{\mathrm{H}+}=\sum_{i=1}^{N} \Lambda_{i} j_{i} .
$$

This last expression is derived exactly as the equivalent of Eq. (12) of Ref. [16]. The ratio of any two of the ion species current densities is

$$
\frac{j_{\alpha}}{j_{\beta}}=\frac{Z_{\alpha} n_{\alpha}}{Z_{\beta} n_{\beta}} \sqrt{\frac{\Lambda_{\beta}}{\Lambda_{\alpha}}},
$$

and this expression is consistent with Eq. (15) of Ref. [16]. Charge and particle conservation in steady state yields

$$
\frac{Z_{\alpha} n_{\alpha}}{Z_{\beta} n_{\beta}}=\frac{k_{\alpha}}{k_{\beta}}
$$

By straightforward algebraic substitutions in Eqs. (C1)(C4) we obtain the summation rule given by Eq. (12) in Sec. VI. The total ion current density is then given by

$$
j=\frac{j_{H+}}{\sqrt{\Lambda}} .
$$

Equation (C5) using the summation rule Eq. (12) is compared to 1D ES CHICAGO simulations in Fig. 10 for mixtures of $\mathrm{H}^{+1}$ and $\mathrm{O}^{+4}$. For these test cases, $V=3 \mathrm{MV}$ and $d=2 \mathrm{~mm}$ as used in Table for the WDC-HE 1D ES cases, except that for Fig. 10 these is no electron space charge in these calculations. For all ion species ratios, the simulations are in excellent agreement with the CL multiple-ion current density given by Eq. (C5). Good agreement is also obtained between the WDC-HE model using the summation rule, and the $1 \mathrm{D}$ ES simulation results listed in Table II for the cases with $B=0$. Although not shown here, we have also compared three ion species simulations to Eq. (C5) and found excellent agreement in all cases.

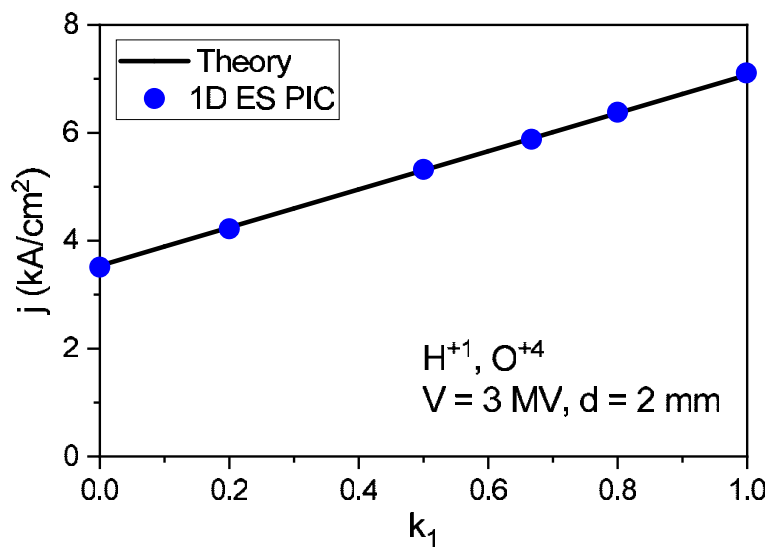

FIG. 10. Multiple ion species current density for $\mathrm{H}^{+1}$ and $\mathrm{O}^{+4}$ ions at $V=3 \mathrm{MV}$ and $d=2 \mathrm{~mm}$. The solid black line is from Eq. (C5) and the blue points are from 1D ES PIC simulations.

\section{APPENDIX D: COMPARISON OF REFS. [5] AND [10] ENHANCEMENT FACTOR FORMULATION}

Equations (8) and (C11) of Ref. [10] express the inner MITL ion loss enhancement factor as

$$
\eta_{[10]}=\frac{3}{4} k_{v i} Q_{e} \frac{d}{\epsilon_{0} V A}
$$

in MKS units. In Eq. (D1), the accumulated electron charge $Q_{e}$ is defined as

$$
Q_{e}=\frac{A_{s}}{A_{T}} f_{e n, i m} \int_{0}^{t} I_{f} d t
$$

where $A_{s}$ and $A_{T}$ are the considered transmission line segment and total inner MITL area, respectively, $f_{e n, i m}$ is the fraction of the inner MITL surviving sheath current from the outer MITLs, $V$ is the instantaneous voltage, and $d$ is the AK gap of that segment. The $k_{v i}$ a numerical factor taken as a best fit to be 1.3. Thus,

$$
\eta_{[10]} \simeq Q_{e} \frac{d}{\epsilon_{0} V A}
$$

On the other hand, the enhancement factor for the HE regime is given in Eq. (14b) in Ref. [5] as

$$
\eta_{[5]}=\frac{9}{16} \gamma_{0}^{2} .
$$

Using the nomenclature and definitions in Ref. [5], we obtain (in MKS units),

$$
\eta_{[5]}=\frac{9}{8} Q_{e \mathrm{HE}} \frac{d}{\epsilon_{0} V A_{s}},
$$

where

$$
\frac{Q_{e \mathrm{HE}}}{A_{s}}=\gamma_{0}\left(\gamma_{0}+\pi\right) \frac{V \epsilon_{0}}{4 d}, \quad \gamma_{0} \geq \pi,
$$

is the electron space charge in a segment of area $A_{s}=$ $2 \pi r \Delta r$ (see HE case Eq. (14) of Ref. [5]), and where (in CGS units and currents in A),

$$
\gamma_{0}^{2} \simeq \frac{2 I_{a} I_{e} d^{2}}{25 r^{2} V^{2}}
$$

is a dimensionless variable expressing the diamagnetic effect of the electron space charge.

Although, formally, Eqs. (D3) and (D5) exhibit the same functional dependence, differing only by a factor $9 / 8$ in Eq. (D5), the manner in which the electron charge depends on the physical variables is indeed distinct. That is in Ref. [10], the ion current enhancement is obtained from the time integral of the fraction of electron space charge, 
dynamically calculated from the sheath current entering from the outer MITLs and convolute, and employing parameters fitted by comparing the code results with numerous $Z$ experiments. In Ref. [5] by contrast, that charge is obtained from solving Poisson's and Ampere's laws in 1D planar steady state as a function of $\gamma_{0}$ [Eq. (D5)]. Therefore the electron charge, and thus the enhancement factor depends explicitly on the anode and electron sheath currents, as well as the voltage and AK gap; variables that can be interpreted in a "snapshot" time dependent manner. Direct numerical comparison would be needed to ascertain the magnitude of the differences in the enhancement factors for various $Z$ operating parameters.

[1] M. R. Gomez, R. M. Gilgenbach, M. E. Cuneo, C. A. Jennings, R. D. McBride, E. M. Waisman, B. T. Hutsel, W. A. Stygar, D. V. Rose, and Y. Maron, Experimental study of current loss and plasma formation in the $Z$ machine post-hole convolute, Phys. Rev. Accel. Beams 20, 010401 (2017).

[2] D. V. Rose, E. A. Madrid, D. R. Welch, R. E. Clark, C. B. Mostrom, W. A. Stygar, and M. E. Cuneo, Computational analysis of current-loss mechanisms in a post-hole convolute driven by magnetically insulated transmission lines, Phys. Rev. ST Accel. Beams 18, 030402 (2015).

[3] R. W. Lemke, D. B. Sinars, E. M. Waisman, M. E. Cuneo, E. P. Yu, T. A. Haill, H. L. Hanshaw, T. A. Brunner, C. A. Jennings, W. A. Stygar et al., Effects of Mass Ablation on the Scaling of X-Ray Power with Current in Wire-Array $Z$ Pinches, Phys. Rev. Lett. 102, 025005 (2009).

[4] C. A. Jennings, M. E. Cuneo, E. M. Waisman, D. B. Sinars, D. J. Ampleford, G. R. Bennett, W. A. Stygar, and J. P. Chittenden, Simulations of the implosion and stagnation of compact wire arrays, Phys. Plasmas 17, 092703 (2010).

[5] E. M. Waisman, M. P. Desjarlais, and M. E. Cuneo, Ion current losses in the convolute and inner magnetically insulated transmission line of the $Z$ machine, Phys. Rev. Accel. Beams 22, 030402 (2019).

[6] R. B. Spielman, C. Deeney, G. A. Chandler, M. R. Douglas, D. L. Fehl, M. K. Matzen, D. H. McDaniel, T. J. Nash, J. L. Porter, T. W. L. Sanford et al., Tungsten wirearray Z-pinch experiments at 200 TW and 2 MJ, Phys. Plasmas 5, 2105 (1998).

[7] D. H. McDaniel, M. G. Mazarakis, D. E. Bliss, J. M. Elizondo, H. C. Harjes, H. C. Ives, D. L. Kitterman, J. E. Maenchen, T. D. Pointon, S. E. Rosenthal et al., The ZR refurbishment project, in Proc. 5th International Conf. on Dense Z-Pinches, edited by J. Davis (American Institute of
Physics, New York, 2002), p. 23, https://doi.org/10.1063/ 1.1531273.

[8] W. A. Stygar, P. A. Corcoran, H. C. Ives, R. B. Spielman, J. W. Douglas, B. A. Whitney, M. A. Mostrom, T. C. Wagoner, C.S. Speas, T.L. Gilliland et al., 55-TW magnetically insulated transmission-line system: Design, simulations, and performance, Phys. Rev. Accel. Beams 12, 120401 (2009).

[9] M. P. Desjarlais, Theory of applied-B ion diodes, Phys. Fluids B 1, 1709 (1989).

[10] B. T. Hutsel, P. A. Corcoran, M. E. Cuneo, M. R. Gomez, M. H. Hess, D. D. Hinshelwood, C. A. Jennings, G. R. Laity, D. C. Lamppa, R. D. McBride et al., Transmissionline-circuit model of an 85-TW, 25-MA pulsed-power accelerator, Phys. Rev. Accel. Beams 21, 030401 (2018).

[11] D. R. Welch, N. Bennett, T. C. Genoni, D. V. Rose, C. Thoma, C. Miller, and W. A. Stygar, Electrode contaminant plasma effects in $10^{7}$-A $Z$ pinch accelerators, Phys. Rev. Accel. Beams 22, 070401 (2019).

[12] N. Bennett, D. R. Welch, C. A. Jennings, E. Yu, D. V. Rose, B. T. Hutsel, G. Laity, K. Peterson, and M. E. Cuneo, Current transport and loss mechanisms in the $Z$ accelerator, Phys. Rev. Accel. Beams 22, 120401 (2019).

[13] C. Thoma, D. R. Welch, R. E. Clark, D. V. Rose, and I. E. Golovkin, Hybrid-PIC modeling of laser-plasma interactions and hot electron generation in gold hohlraum walls, Phys. Plasmas 24, 062707 (2017).

[14] R. E. Clark, D. R. Welch, W. R. Zimmerman, C. L. Miller, T. C. Genoni, D. V. Rose, D. W. Price, P. N. Martin, D. J. Short, A. W. P. Jones et al., Locally conformal finitedifference time-domain techniques for particle-in-cell plasma simulation, J. Comput. Phys. 230, 695 (2011).

[15] M. S. Di Capua and D. G. Pellinen, Propagation of power pulses in magnetically insulated vacuum transmission lines, J. Appl. Phys. 50, 3713 (1979).

[16] S. Qin, Z. Jin, and C. Chan, Dynamic sheath model of collisionless multispecies plasma immersion ion implantation, J. Appl. Phys. 78, 55 (1995).

[17] E. A. Madrid, D. V. Rose, D. R. Welch, R. E. Clark, C. B. Mostrom, W. A. Stygar, M. E. Cuneo, M. R. Gomez, T. P. Hughes, T. D. Pointon et al., Steady-state modeling of current loss in a post-hole convolute driven by high power magnetically insulated transmission lines, Phys. Rev. Accel. Beams 16, 120401 (2013).

[18] D. V. Rose, T. C. Genoni, R. E. Clark, D. R. Welch, and W. A. Stygar, Electron flow stability in magnetically insulated vacuum transmission lines, Phys. Plasmas 18, 033108 (2011).

[19] D. V. Rose, D. R. Welch, T. P. Hughes, R. E. Clark, and W. A. Stygar, Plasma evolution and dynamics in high-power vacuum-transmission-line post-hole convolutes, Phys. Rev. Accel. Beams 11, 060401 (2008). 